\title{
Epinephrine versus tramazoline to reduce nasal bleeding during nasotracheal intubation. A double- blind randomized trial
}

Aiji Sato(Boku) ( $\square$ bokuaiji@dpc.agu.ac.jp )

Aichi Gakuin University

Yoshiki Sento

Nagoya City University

Yuji Kamimura

Nagoya City University

Eisuke Kako

Nagoya City University

Masahiro Okuda

Aichi Gakuin University

Naoko Tachi

Aichi Gakuin University

Yoko Okumura

Aichi Gakuin University

Mayumi Hashimoto

Aichi Gakuin University

Hiroshi Hoshijima

Tohoku University

Fumihito Suzuki

Akita National Hospital

Kazuya Sobue

Nagoya City University

\section{Research Article}

Keywords: Nasotracheal intubation, Epinephrine, Tramazoline, Nasal bleeding

Posted Date: February 10th, 2021

DOI: https://doi.org/10.21203/rs.3.rs-148779/v1 
License: (c) (i) This work is licensed under a Creative Commons Attribution 4.0 International License. Read Full License 


\section{Abstract}

BACKGROUND $\triangle$ Nasal bleeding is the most common complication during nasotracheal intubation (NTI). To reduce nasal bleeding, the nasal cavity is treated with vasoconstrictors (epinephrine [E] or tramazoline $[T]$ ) prior to NTI. This study aimed to determine whether $\mathrm{E}$ or $\mathrm{T}$ is more effective and safe for reducing nasal bleeding during $\mathrm{NTI}$.

METHODS $\triangle$ This study was preregistered on UMIN-CTR after being approved by the IRB of the School of Dentistry at Aichi Gakuin University. Written consent was received from all the patients. Total 206 patients aged 20-70 years and classified as 1-2 on American Society of Anesthesiologists-physical status were scheduled to undergo general anesthesia with NTI. Patients with a narrowed nasal cavity observed during preoperative CT test $(n=3)$, patients with hypertension $(n=3)$, patients undergoing antithrombotic therapy, and patients who did not give consent $(n=3)$ were excluded from the study. At last, 197 patients were randomly divided into two groups and treated with either $E(n=99 ; 3$ patients were discontinued) or $T$ ( $n=98 ; 2$ patient were discontinued). After induction of general anesthesia, each patient's nasal cavity was treated using either E or T. Next, NTI was performed via the right nostril. Primary outcome was the presence of nasal bleeding. If bleeding was recognized at the posterior pharyngeal wall via nasal cavity during intubation, it was defined as bleeding. Secondary outcomes were the degree of bleeding (classified as none, mild, moderate, or severe), arrhythmia, and hemodynamic (mean atrial pressure and heart rate) changes associated with vasoconstrictors.

RESULTS $₫$ The presence of bleeding was comparable in both groups $(12.5 \%, E ; 14.5 \%, T ; P=0.63)$. No significant difference between the groups regarding the degree of bleeding $(P=0.78)$ was observed, with most patients having no bleeding $(n=84, E ; n=82, T)$. No severe bleeding and no arrhythmias induced by vasoconstrictor were observed in the two groups.

CONCLUSIONS $₫$ Nasal treatment with E or T shows no difference in nasal bleeding during NTI. Although no arrhythmia associated with E was observed in this study, it has been reported in literature. Therefore, as frequency and degree of nasal bleeding were comparable, nasal treatment with $T$ could reduce the risk of NTI.

Trial Registration

UMIN-CTR (Registration No. UMIN000037907)

\section{Background}

Nasotracheal intubation (NTI) is frequently necessary during dental, maxillofacial, and oropharyngeal surgeries. Although this method is very useful in operations wherein the operative field and airway are congruent, some complications associated with NTI include nasal bleeding [1, 2], bacteremia [3], retropharyngeal perforation [4], and partial or complete obstruction of the tube [5,6] has been reported. Several effective preventive measures against nasal bleeding, retropharyngeal perforation, and 
bacteremia have been reported $[7,8,9,10]$. Previous studies $[11,12,13]$ have also explored different hemostatic agents used during NTI; however, the type of vasoconstrictor is still debatable. The nasal cavity is usually treated with vasoconstrictors, such as epinephrine (E) or tramazoline (T), prior to NTI. T has been used for treatment since 1960 and E even before that. Considering their significant use in medical procedures for $>60$ years, their efficacy and side effects are well known. Although arrhythmia has been reported in literature for nasal treatments performed with E [14], no previous reports compared the vasoconstriction effects and safety of these two vasoconstrictors during NTI. This study aimed to determine whether $\mathrm{E}$ or $\mathrm{T}$ is more effective and safe for reducing nasal bleeding during NTI.

\section{Methods}

\section{Ethics approval and consent to participate}

The study was performed in accordance with the ethical standards of the Declaration of Helsinki (1964) and its subsequent amendments. This study was adhered to CONSORT guidelines. This study was approved by the Ethics Committee at the School of Dentistry, Aichi Gakuin University (Approval No. 566), and written informed consent was obtained from all subjects participating in the trial. Prior to patient enrollment, the trial was registered as a clinical trial at UMIN-CTR (Registration No. UMIN000037907, Date of first registration: 05/09/2019). The first patient was recruited and registered on 4th October, 2019.

\section{Study design and population}

We conducted a prospective, double-blind randomized study, enrolling 206 patients aged $20-70$ years and classified into classes 1 and 2 as per American Society of Anesthesiologists-physical status that were scheduled to undergo general anesthesia with NTI. Among them, patients with a narrowed nasal cavity observed during preoperative CT test $(n=3)$, patients with hypertension and using antihypertensive drugs $(n=3)$, and patients undergoing antithrombotic therapy $(n=0)$ were excluded. In addition, patients who did not give consent $(n=3)$ were excluded from the study. Therefore, the final study population included 197 patients who were randomly divided into two groups: the group treated with $E(n=99)$ and the group treated with $T(n=98)$. An anesthetist who was not involved in the study conducted the randomization in accordance with a computer-generated random number. The participant flow diagram is presented in Fig. 1.

\section{Anesthesia and intubation methods}

The same method of anesthesia was employed for all patients. No premedication was administered. After a patient walked independently to the operating theater, the standard vital signs monitors (electrocardiogram, blood pressure, and oxygen saturation) were inspected. Anesthesia was induced using propofol ( $4 \mu \mathrm{g} / \mathrm{ml}$ target control infusion), remifentanil $(0.2 \mu \mathrm{g} / \mathrm{kg} / \mathrm{min})$, and fentanyl $(100 \mu \mathrm{g})$ with rocuronium $(0.6 \mathrm{mg} / \mathrm{kg})$ used as a neuromuscular blocking agent. Until the effects of the neuromuscular blocking agent became apparent, mask ventilation was applied in all patients using $100 \%$ oxygen with propofol and remifentanil. While mask ventilation was being performed, the subjects' nasal cavities and 
inferior nasal passages were adequately disinfected with BZK spray (ZALKONIN® SOLUTION 0.025, Kenei Phamaceutical Co., Ltd, Osaka). During disinfection, swabbing was not done to avoid nasal bleeding. Next, we soaked a $10 \mathrm{~cm} \times 3 \mathrm{~cm}$ gauze in $2 \mathrm{ml}$ of randomly pre-specified chemical solution (E or $\mathrm{T})$. Next, the gauze was inserted through the nasal cavity into the lower nasal passage using a rhino scope and louche (Fig. 2). After 2 min, the gauze was removed and NTI was performed in the right nostril [7]. The E used in this study was BOSMIN® SOLUTION 0.1\% (Daiichi-Sankyo Co., Ltd, Tokyo), and the T used in this study was TRAMAZOLIN Nasal Solution 0.118\%ロAFP区, (Alfresa Pharma Corporation, Osaka). $E$ was diluted five times according to the package insert (final concentration of $E=0.02 \%$ ), and $T$ was used in its original solution. The intubation tube used in this study was Polar ${ }^{\mathrm{TM}}$ Preformed Tracheal Tube (Smith Medical Japan Ltd, Tokyo), and the tube size was ID 7.0 for males and ID 6.5 for females.

\section{Measurements}

The primary outcome was the presence or absence of nasal bleeding observed at the posterior pharyngeal wall during intubation. The secondary outcomes were the degree of bleeding and presence or absence of arrhythmia associated with vasoconstrictors detected through an electrocardiography. The degree of bleeding was graded on four levels (none, mild, moderate, and severe), determined as follows: none-no bleeding on the posterior wall of the pharynx at the time of intubation and 10 min after intubation, and no bleeding from the nasal cavity at extubation; mild-bleeding in the posterior wall of the pharynx at intubation, no bleeding in the posterior wall of the pharynx 10 min after intubation, and no bleeding from the nasal cavity at extubation; moderate-bleeding on the posterior wall of the pharynx at the time of intubation and 10 min after intubation, but no bleeding from the nasal cavity at extubation; severe-bleeding on the posterior wall of the pharynx at the time of intubation and 10 minutes after intubation, and bleeding from the nasal cavity at extubation. In addition, mean arterial pressure (MAP) and heart rate (HR) was measured before and after the use of vasoconstrictors. The years of experience of the intubator was also collected.

\section{Statistical analysis}

Based on our estimation, a minimum sample of 178 patients ( $n=89$ per group), where the effect size, minimal significance $(\alpha)$ and statistical power $(1-\beta)$ were set at $0.22,0.05$, and 0.80 respectively. The effect size was calculated on the basis of the statistical results of a pilot study wherein the patient distribution in the number of nasal bleeding after NTI was used as a standard ( $E$ group, $n=10 ; T$ group, $n$ $=10$ ). As the use of statistical tests in the absence of reliable sample size calculation decreases its weightage, we calculated our final sample size considering an expected dropout rate of 0.05 based on our pilot study. Hence, if a dropout rate $(R)$ is expected, a simple but adequate adjustment is provided by $N_{d}=$ $\mathrm{N} /(1-\mathrm{R})^{2}$, where $\mathrm{N}$ is the sample size calculated assuming no dropout and $\mathrm{N}_{d}$ is the estimated sample size required when dropouts are expected [15]. Therefore, after adjusting for dropouts, a final sample of 197 patients was observed. For statistical testing, Chi-Square test of independence was used to test for a relationship between two categorical variables, and the Mann-Whitney $U$ test was used to compare differences between two independent groups for continuous variables. Wilcoxon signed-rank test was 
used to compare differences between two dependent groups for continuous variables. The two-sided statistical significance level was set at $p \leq 0.05$.

\section{Results}

From October 2019 to September 2020, 206 patients were selected to participate in this study. The CONSORT diagram of patient flow through the study is illustrated in Fig. 1. From the 197 subjects randomly assigned into two groups on the basis of the vasoconstriction method used ( $E$ or $T$ ), 5 subjects dropped out during the trial. The final analytical sample included 192 patients (96 per group).

Demographic characteristics, operation and anesthesia time, and the years of experience of the intubator are presented in Table I.

No statistical differences between the groups were observed. The presence of bleeding was observed in 12 patients from the $E$ group and in 14 patients from the $T$ group, representing a similar proportion in the two groups $(12.5 \%$ vs. $14.5 \%, P=0.63)$. No significant difference between the groups $(P=0.78)$ regarding the degree of bleeding was observed (Table II).

Table III shows the change in hemodynamics with the use of vasoconstrictors. In group E, the MAP significantly increased after the vasoconstrictor was applied (mean, $71.5 \mathrm{mmHg}$ vs. $80.0 \mathrm{mmHg}, \mathrm{P}=$ 0.001), whereas no significant differences were observed in the HR (mean, 64.5 beat/min vs. 65.0 beat/min $P=0.24)$. Likewise, in group $T$, the MAP significantly increased after the vasoconstrictor was applied (mean, $72 \mathrm{mmHg}$ vs. $77 \mathrm{mmHg}, \mathrm{P}=0.04$ ). In contrast, the HR remained stable during the NTI procedure (mean, 68.5 beat $/ \mathrm{min}$ vs. 68.0 beat $/ \mathrm{min}, \mathrm{P}=0.91$ ).

No arrhythmias induced by vasoconstrictor use were observed in the two groups.

\section{Discussion}

In this study, using E or T as a vasoconstrictor, we observed no difference in the frequency and degree of bleeding between the groups. Factors influencing the frequency of bleeding may include the patient's sex and the years of experience of the intubator. However, in this study, both groups had similar characteristics, which is ideal for proper investigation if the potential bleeding occurrence was only associated with the vasoconstrictor.

Nasal bleeding due to NTI is mostly caused by damaging of the venous plexus in the middle nasal mucosa, the curvature of the nasal septum, and adenoid enlargement [16]. In addition, nasal bleeding during NTI can obstruct the airway patent and cause serious complications such as airway obstruction. Considering that persistent bleeding can also affect the operative field, every effort should be made to prevent bleeding whenever possible. Vasoconstrictors are used to achieve local hemostasis by constricting the intramucosal capillaries and reducing blood flow, thereby expanding the nasal volume and facilitating the passage of the intubation tube through the nasal cavity [16]. 
Epinephrine is the most common vasoconstrictor for local hemostatic purposes, with recommended concentrations of $0.1-0.02 \%$ [17]. Previous studies $[11,18]$ using $E$ to prevent nasal bleeding reported hemorrhage rates of $37 \%$ [11] and $60 \%$ [18], which are considerably higher than the $12.5 \%$ of nasal bleeding observed in the present study. This discrepant finding may be attributed to the concentration of E, nasal stenosis, and left-right differences in the nasal cavity used. Note that both previous studies [11, 18] used an E concentration of $0.01 \%$, whereas in our study we used a concentration of $0.02 \%$. Although some blood pressure fluctuation was observed before and after use of $\mathrm{E}$, it was not clinically problematic, and the $0.02 \%$ concentration could be used without problems. Furthermore, $E$ has an onset of action of 5 min and lasts for $1 \mathrm{~h} \mathrm{[18].} \mathrm{For} \mathrm{practical} \mathrm{reasons,} \mathrm{we} \mathrm{set} \mathrm{a} \mathrm{time} \mathrm{of} 2 \mathrm{~min}$ (instead of $5 \mathrm{~min}$ ) and we found that a 2-min period was sufficient to achieve hemostasis.

In this study, as patients were undergoing dental surgery, a computed tomography of the facial area was taken in all cases prior to the study. Therefore, it was possible to include nasal stenosis as an exclusion criterion for the current study. Hence, despite the 4-year average intubation experience, the passage of the intubation tube was smooth and performed at once in all patients. Moreover, all patients were intubated using the right nasal cavity, as we have previously reported less epistaxis in the right nasal cavity compared with the left nasal cavity [7]. Unlike the current study, Sonan et al [18] used the left nasal cavity for intubation, but it is unclear which nasal cavity was used in the study by Jaegyok et al.[11] We believe that the above three factors contributed to the lower percentage of bleeding observed in our study than in the previous studies $[11,18]$.

In contrast, we also used T as a vasoconstrictor in this study. T stimulates alpha-2 receptors and constricts local blood vessels in the nasal cavity, eliminating hyperemia and swelling of the nasal mucosa [19]. There are reports that nasal T infusion improves rhinitis [20] and sleep apnea [21]. To the best of our knowledge, there are no reports regarding use of $\mathrm{T}$ for preventing nasal bleeding during NTI. Our findings indicate that $T$ is as effective as $E$ regarding prevention of nasal bleeding during NTI. The blood pressure fluctuation observed in the present study during the use of both drugs is due to the sympathetic stimulating effects of both drugs. Although the blood pressure fluctuations in both groups were clinically safe, $E$ increased blood pressure more than $T$, suggesting that $E$ stimulates alpha receptors more strongly than $\mathrm{T}$ within the concentrations used in this study. Although patients with hypertension were excluded from the study, the use of both drugs increased blood pressure of normotensive patients, suggesting the need for extremely careful judgment while using these drugs in hypertensive patients.

In the present study, we also found that the use of both drugs did not induce arrhythmias. It has also been reported that the use of $E$ for the prevention of nasal bleeding usually has a minimal effect on circulation in many patients $[22,23]$. However, sudden tachycardia was reported when E was used to prevent nasal bleeding [14]. In that study, it was assumed that severe tachycardia was triggered by submucosal migration of an epinephrine-soaked swab. Hence, even at normal concentrations of $E$, when the mucous membrane is injured by swabs or other means, it is important to consider that a rapid increase of concentration of $E$ in the blood may induce arrhythmia. In the present study, total intravenous anesthesia was used in all patients. However, previous studies reported a decrease in the arrhythmia range of $E$ in 
combination with volatile anesthetics with halogenated anesthetics ranging from halothane to sevoflurane $[24,25]$. Therefore, attention should be given to variations in circulation dynamics while using E.

The major limitation of this study is the exclusion of patients with nasal stenosis; different results could be expected if patients with nasal stenosis were included. However, mixing of patients with and without nasal stenosis in the same trial must be avoided. This issue should be investigated in future studies.

In conclusion, nasal treatment with E or T shows no difference in nasal bleeding during NTI. Although no arrhythmia associated with $\mathrm{E}$ was observed in this study, it has been reported in the literature. Therefore, as the frequency and degree of nasal bleeding were comparable, nasal treatment with $T$ could reduce the risk of NTI.

\section{Abbreviations}

NTI: Nasotracheal intubation

E: Epinephrine

T: Tramazoline

MAP: Mean arterial pressure

HR: Heart rate

\section{Declarations}

Ethics approval and consent to participate. $3 / 4$ This study was performed in accordance with the ethical standards of the Declaration of Helsinki (1964) and its subsequent amendments. This study was approved by the Ethics Committee at the School of Dentistry, Aichi Gakuin University (Approval No. 566) and written informed consent was obtained from all subjects participating in the trial.. Prior to patient enrollment, the trial was registered as a clinical trial at UMIN-CTR (Registration No. UMIN000037907, Principal investigator: Aiji Sato, Date of registration: September 5, 2019). Our study adhered to CONSORT guidelines. The first patient was recruited and registered on October 4, 2019.

Consent for publication.3/4 Not applicable.

Availability of data and materials. $3 / 4$ The datasets analyzed during the current study are available from the corresponding author on reasonable request.

Competing interest. $3 / 4$ The authors certify that there is no competing interest with

any financial organization regarding the material discussed in the manuscript. 
Funding.3/4 The authors report no involvement in the research by the sponsor that could have influenced the outcome of this work.

Authors' contributions. $3 / 4$ AS helped with substantial contribution to the conception or design of the work, statistics, planning, codunt, reporting of the work, and write the manuscript. YS helped with substantial contribution to the conception or design of the work and interpret the study results. YK helped with substantial contribution to the conception or design of the work and interpret the study results. EK helped with substantial contribution to the conception or design of the work and interpret the study results. MO helped with substantial contribution to the conception or design of the work, conduct and reporting of the work. YO helped with substantial contribution to the conception or design of the work, conduct and reporting of the work. NT helped with substantial contribution to the conception or design of the work, conduct and reporting of the work. MH helped with substantial contribution to the conception or design of the work and interpret the study results. $\mathrm{HH}$ helped with substantial contribution to the conception or design of the work, statistics and write the manuscript. FS helped with substantial contribution to the conception or design of the work, statistics and write the manuscript. KS helped with substantial contribution to the conception or design of the work, supervision of the manuscript and development of the overall study.

Acknowledgements. $3 / 4$ The authors would like to thank Toshiyuki Nakanishi, M.D, Department of Anesthesiology and Intensive Care Medicine, Nagoya City University Graduate School of Medical Sciences (Nagoya, Japan) for the advice on the manuscript and all of the participants for their cooperation in the study. The authors would like to thank Enago for manuscript review and editing support.

\section{References}

1. Lee JH, Kim CH, Bahk JH, Park KS. The influence of endotracheal tube tip design on nasal trauma during nasotracheal intubation: Magill-tip versus Murphy-tip. Anesth Analg 2005;101:1226-9.

2. Chen YH, Chen JY, Hsu CS, Huang CT, So E. Recurrent epistaxis following nasotracheal intubation: a case report. Acts Anaesthesiol Sin 1996;34:93-6.

3. Berry FA, Blankenbaker WL, Ball CG. Comparison of bacteremia occurring with nasotracheal and orotracheal intubation. Anesth Analg 1973;52:873-6.

4. Chait DH, Poulton TJ. Case report: retropharyngeal perforation, a complication of nasotracheal intubation. Nebr Med J 1984;69:68-9.

5. Kenney JN, Laskin DM. Nasotracheal tube obstruction from a central incisor. Report of a case. Oral Surg Oral Med Oral Pathol 1989;67:266-7.

6. Tintinalli JE, Claffey J. Complications of nasotracheal intubation. Ann Emerg Med 1981;10:142-4.

7. Boku A, Hanamoto H, Hirose Y, Kudo C, Norimoto Y, Sugimura M, et al. Which nostril should be used for nasotracheal intubation: the right or left? A randomized clinical trial. J Clin Anesth 2014;26:3904. 
8. Sanuki T, Hirokane M, Matsuda Y, Sugioka S, Kotani J. The Parker Flex-Tip tube for nasotracheal intubation: the influence on nasal mucosal trauma. Anaesthesia 2010;65:8-11.

9. Morimoto Y, Sugimura M, Hirose Y, Taki K, Niwa H. Nasotracheal intubation under curve-tipped suction catheter guidance reduces epistaxis. Can J Anesth 2006;53:295-8.

10. Sato-Boku A, Nagano K, Hasegawa Y, Kamimura Y, Sento Y, So M, Kako E, Okuda M, Tachi N, Ito H, Adachi Y, Sobue K. Comparison of disinfection effect between benzalkonium chloride and povidone iodine in nasotracheal intubation: a randomized trial. BMC Anesthesiol 2019;19:168.

11. Song J. A comparison of the effects of epinephrine and xylometazoline in decreasing nasal bleeding during nasotracheal intubation. J Dent Anesth Pain Med 2017;17:281-7.

12. Han JS, Park SH, Shin YD, Kwon E, Shim WS, Bae JH. The effect of xylometazoline spray for expansion of nasal cavity. Korean J Anesthesiol 2013;65:132-5.

13. El-Seify ZA, Khattab AM, Shaaban AA, Metwalli OS, Hassan HE, Ajjoub LF. Xylometazoline pretreatment reduces nasotracheal intubation-related epistaxis in paediatric dental surgery. Br J Anaesth 2010;105:501-5.

14. Hoshi T, Suzuki T, Somei M, lijima T, Kurihara Y. Sudden tachycardia due to submucosal migration of an epinephrine-soaked swab during nasal intubation. Anesth Prog 2018;65:259-60.

15. Lachin JM. Introduction to sample size determination and power analysis for clinical trials. Control Clin Trials 1981;2:93-113.

16. Ota S, Yoshikawa M, Syouji K, Tanifuji Y. Comparison of epinephrine and phenylephrine concentrations for use during intranasal procedures. Oto-Rhino Laryngol 2006;49:360-364.

17. Nolan RT. Nasal intubation. An anatomical difficulty with Portex tubes. Anaesthesia 1969;24:447-8.

18. Patel S, Hazarika A, Agrawal P, Jain D, Panda NK. A prospective randomized trial of xylometazoline drops and epinephrine merocele nasal pack for reducing epistaxis during nasotracheal intubation. $J$ Dent Anesth Pain Med 2020;20:223-1.

19. Langer SZ. Presynaptic regulation of the release of catecholamines. Pharmacol Rev 1980;32:33762.

20. Katona G, Sultész M, Farkas Z, Gyimesi A, Hirschberg A, Huszka J, Radvánszky F, Simon Á, Fülöp G, Láng J, Ablonczy M, Nirnberger G, Holm C. Treatment of acute rhinitis with a nasal spray containing tramazoline and essential oils: a multicenter, uncontrolled, observational trial. Clin Transl Allergy 2015;5:38.

21. Koutsourelakis I, Minaritzoglou A, Zakynthinos G, Vagiakis E, Zakynthinos S. The effect of nasal tramazoline with dexamethasone in obstructive sleep apnoea patients. Eur Respir J 2013;42:105563.

22. Katz RI, Hovagim AR, Finkelstein HS, Grinberg Y, Boccio RV, Poppers PJ. A comparison of cocaine, lidocaine with epinephrine, and oxymetazoline for prevention of epistaxis on nasotracheal intubation. J Clin Anesth 1990;2:16-20. 
23. Kameyama K, Watanabe S, Kano T, Kusukawa J. Effects of nasal application of an epinephrine and lidocaine mixture on the hemodynamics and nasal mucosa in oral and maxillofacial surgery. $\mathrm{J}$ Oral Maxillofac Surg 2008;66:2226-32.

24. Johnston RR, Eger El, II, Wilson C. A comparative interaction of epinephrine with enfulrane, isofulrane, and halothane in man. Anesth Analg 1976;55:709-12.

25. Hayashi Y, Sumikawa K, Tashiro C, Yamatodani A, Yoshiya I. Arrhythmogenic threshold of epinephrine during sevoflurane, enflurane, and isoflurane anesthesia in dogs. Anesthesiology 1988;69:145-7.

\section{Tables}

Table I. P Patient's demographic characteristics and NTI related information Group E Group T P value

\begin{tabular}{cccc}
\hline \hline Male/Female & $38 / 58$ & $41 / 55$ & 0.65 \\
Age (years) & $32(25-44)$ & $30(25-46)$ & 0.89 \\
\hline Hight (cm) & $162(156-169)$ & $164(158-170)$ & 0.31 \\
\hline Weight (kg) & $55(49-61)$ & $57(50-68)$ & 0.11 \\
\hline Operation time (min) & $75(47-125)$ & $90(69-149)$ & 0.08 \\
\hline Anesthesia time (min) & $127(91-185)$ & $136(103-206)$ & 0.18 \\
\hline Experience of intubator (years) & $4(1-6)$ & $4(1-6.25)$ & 0.98 \\
\hline
\end{tabular}

Values are $\mathrm{n}$ or median (quartile1-quartile3).

Table II. $\square$ Patient's distribution of degree of bleeding 
Group E Group T P value

\begin{tabular}{cccc}
\hline \hline None & 84 & 82 & 0.78 \\
Mild & 10 & 10 & \\
Moderate & 2 & 4 & \\
Severe & 0 & 0 & \\
& & & \\
\hline
\end{tabular}

Values are $n$.

Table III. Hemodynamics before and after the use of vasoconstrictors

$$
\text { Before After P value }
$$

\begin{tabular}{llll}
\hline \hline MAP (mmHg) group E & $71.5(72-90)$ & $80(66-82.5)$ & 0.001
\end{tabular}

HR (beat/min) group E $64.5(58-72) \quad 65$ (60-79) $\quad 0.24$

MAP (mmHg) group T 72 (63-82.5) 77 (69-87.5) $\quad 0.04$

HR (beat/min) group T 68.5 (57.75-78) 68 (58.75-79) 0.91

Values are median (quartile1-quartile3).

MAP, mean atrial presure; HR, heart rate

\section{Figures}




\section{Enrollment}

Assessed for eligibility $(\mathrm{n}=206)$

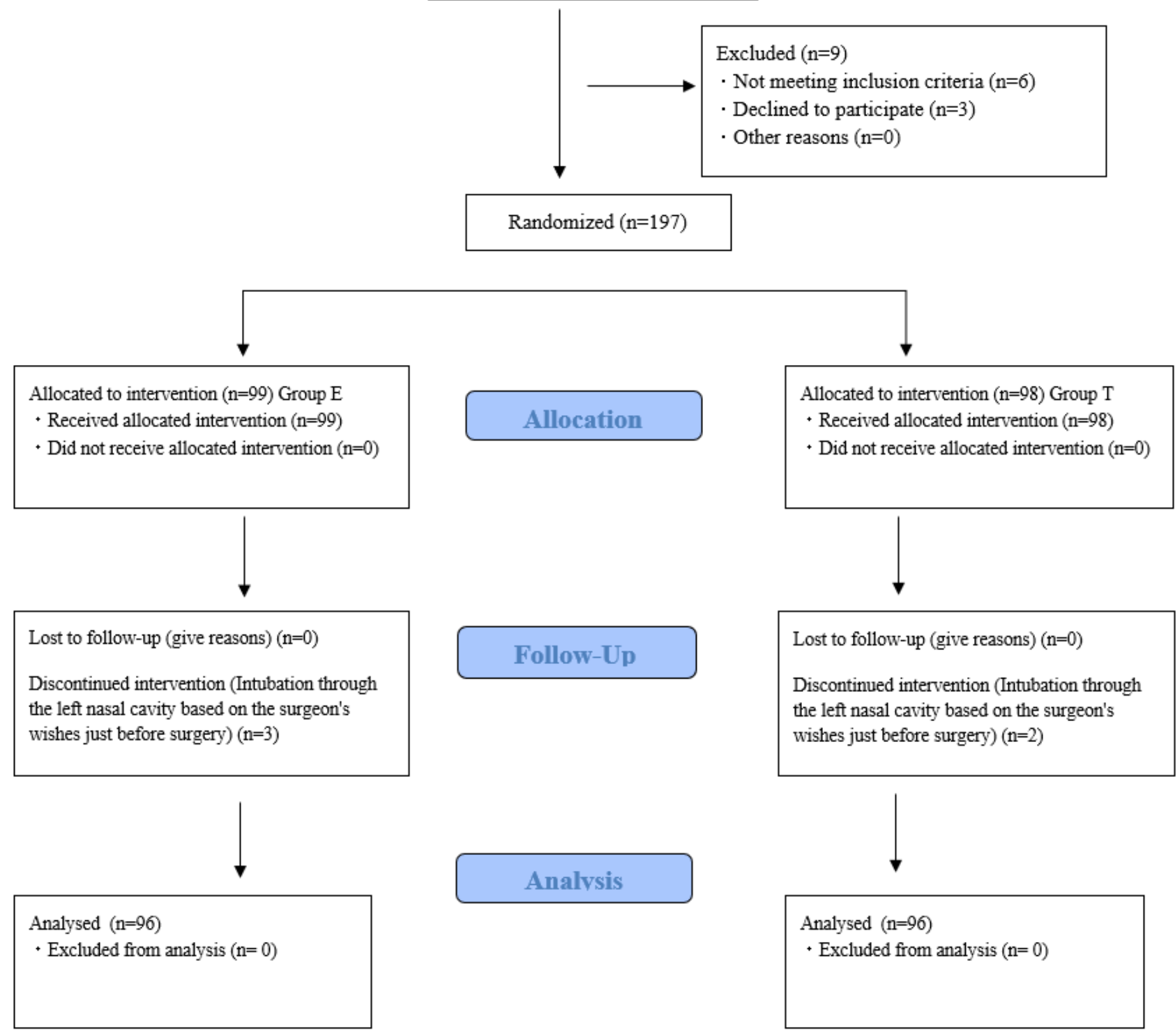

\section{Figure 1}

CONSORT diagram recommended description of patient flow through the study recruitment. 


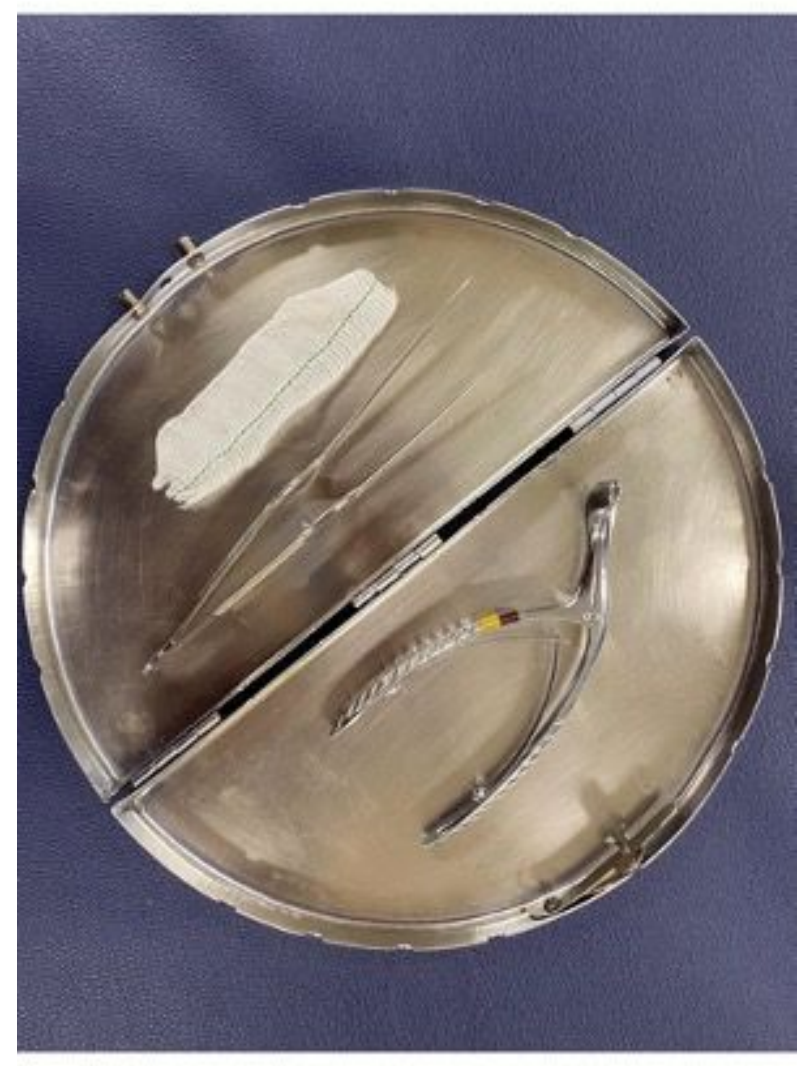

\section{Figure 2}

A $10 \mathrm{~cm} \times 3 \mathrm{~cm}$ gauze in $2 \mathrm{ml}$ of pre-specified chemical solution (E or T), a rhino scope and a louche.

\section{Supplementary Files}

This is a list of supplementary files associated with this preprint. Click to download.

- CONSORT2010ChecklistMSWord.doc 